\section{Botchwey, Dubourdieu, Jia, Kennett, and Park to chair 2018 MRS Spring Meeting}

www.mrs.org/spring2018

$\mathrm{T}^{\mathrm{n}, \mathrm{w}}$

Meeting chairs for the 2018

Materials Research Society (MRS)

Spring Meeting are Edward Botchwey (Georgia Institute of Technology and Emory University, USA), Catherine Dubourdieu (Helmholtz Zentrum Berlin für Materialien und Energie, Germany), Quanxi Jia (University at Buffalo, The State University of New York, USA), Shane Kennett (Exponent Failure Analysis Associates, USA), and Cheolmin Park (Yonsei University, South Korea). The Meeting will be held April 2-6, 2018, in Phoenix, Ariz.

Edward Botchwey is an associate professor at the Wallace H. Coulter Department of Biomedical Engineering at

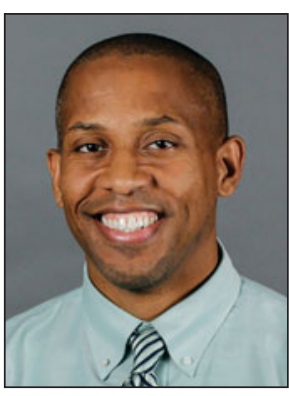

Georgia Institute of Technology and Emory University. He received his BS degree in mathematics from the University of Maryland, College Park. He earned his mas-

ter's degree in materials science engineering and $\mathrm{PhD}$ degree in bioengineering, both from the University of Pennsylvania. He was a postdoctoral scholar at the Wistar Institute in the Division of Vascular Biology.

Botchwey's research focuses on affinity-based controlled release systems to deliver biologically inspired small molecules to control trafficking of blood stem and progenitor cells in vivo as well as the growth and remodeling of microvascular networks. His laboratory is specifically focused on immunoengineering strategies involving bioactive lipid delivery to control trafficking of mononuclear phagocytes and their contributions to angiogenesis, wound healing, and immune tolerance. Previous recognition for his research and leadership includes the Presidential Early Career Award for
Scientists and Engineers. He is a board member of the Biomedical Engineering Society, the immediate past chair of the Gordon Research Conference for Biomaterials and Tissue Engineering, and present chair-elect of the South Eastern Regional Lipid Conference.

Catherine Dubourdieu is the head of the Institute Functional Oxides for Energy-Efficient Information Technology

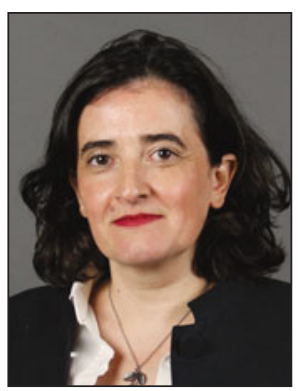
at the Helmholtz Zentrum Berlin für Materialien und Energie (HZB). She is also involved in establishing the Helmholtz Energy Materials Foundry. She earned an engineer diploma and a MS degree from Grenoble Polytechnic Institute in 1992, and a $\mathrm{PhD}$ degree in physics from Grenoble I University in 1995. After a postdoctoral fellowship at Stevens Institute of Technology, USA, she held a permanent position at Centre National de la Recherche Scientifique (CNRS), France, from 1997 to 2016. She co-founded the European School on Nanoscience and Nanotechnology, France, and served as its adjunct director until 2006. From 2009 to 2012, she was a visiting scientist at the IBM T.J. Watson Research Center, USA. From 2013 to 2016, she co-founded and directed the French research network dedicated to functional oxides. In the past 15 years, she has also developed several long-term collaborations with industries and directed a joint laboratory. She moved to HZB in April 2016.

Dubourdieu's research interests include the synthesis and property investigation of oxide thin films and nanostructures, ferroelectricity at the nanoscale, and heteroepitaxy and interfaces on semiconductors. She has more than 130 publications, has delivered 40 invited talks, and holds 10 patents. She was the recipient of a CNRS Bronze Medal in 2001 and an IBM Faculty Award in 2014. She is a member of the International Technology Roadmap for Semiconductors and also serves on the Technical Committees of the Workshop on Dielectrics in Microelectronics and the IEEE Semiconductor Interface Specialists Conference.

Quanxi Jia is an Empire Innovation Professor and National Grid Professor of Materials Research at the University

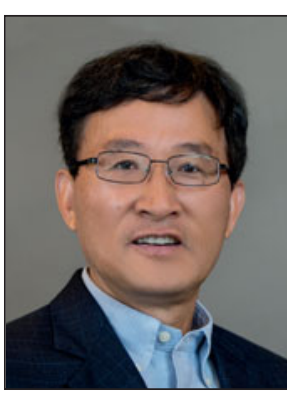
at Buffalo (UB), The State University of New York. He is also the scientific director of the New York State Center for Excellence in Materials Informatics. He received his $\mathrm{BS}$ and MS degrees in electrical engineering from Xi'an Jiaotong University, China, and his $\mathrm{PhD}$ degree in the same field from UB. Previously, he was the director of the Center for Integrated Nanotechnologies, a US Department of Energy Nanoscale Science Research Center operated jointly by Los Alamos and Sandia National Laboratories.

Jia's research areas include synthesis and study of the structure-property relationships of nanostructured materials, multifunctional materials, and thin films; development of novel deposition techniques for the growth of electronic materials; and development and fabrication of novel solid-state microelectronic/ electro-optic devices. He has published more than 450 articles, has delivered more than 100 invited talks, and holds 48 patents. He serves as the co-editor-inchief of the journal Materials Research Letters and sits on the editorial board of several other journals. Among his awards and honors are two R\&D 100 Awards, the 2005 Asian-American Engineer of the Year Award, and the Federal Laboratory Consortium for Technology Transfer Awards for Excellence in Technology Transfer. He is a Fellow of Los Alamos 
National Laboratory, Materials Research Society, American Physical Society, The American Ceramic Society, and American Association for the Advancement of Science.

Shane Kennett is a managing engineer for the consulting firm Exponent Failure Analysis Associates. He con-

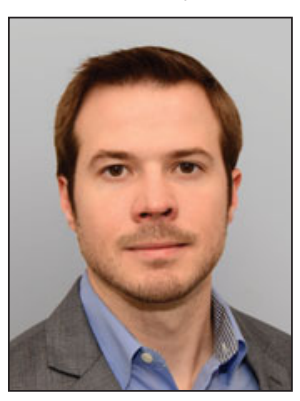
ducted his $\mathrm{PhD}$ degree dissertation work in the Advanced Steels Processing and Products Research Center at the Colorado School of Mines, where he studied

the effects of the martensitic packet size, block size, lath size, dislocation density, and alloy precipitation on the mechanical properties of martensitic steel. Outside of his primary focus, he also studied the mechanical properties of Co-Cr-Mo hip implants, ferrite-pearlite steels, carburized steels, and cold-sprayed copper and aluminum systems.

At Exponent, Kennett specializes in failure analysis, failure prevention, mechanical metallurgy, and fracture mechanics. He studies materials characterization with a strong focus on failure analysis, mechanical behavior of engineering alloys, product life assessment, and materials selection in product design. His work is highly focused in the commercial sector with an emphasis on product liability litigation matters, from which he services a wide range of applications and industries, including surgical devices, medical implants, microelectronics, oil and gas pipelines and refineries, automotive safety equipment, off-road vehicles, and raw material production. $\mathrm{He}$ also has experience consulting for the design and implementation of custom machines used for unique testing scenarios. He also researches the effects of processing on the microstructure and subsequent mechanical properties of different commercial grades of steel.

Cheolmin Park is the Underwood Distinguished Professor in the Department of Materials Science and

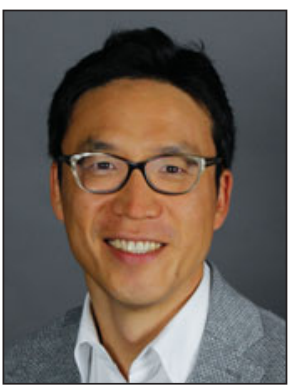

Engineering at Yonsei University. He received his BS degree in 1992 and MS degree in 1995 , both in the Department of Polymer and Fiber Engineering at Seoul National University, South Korea. He received a $\mathrm{PhD}$ degree in the
Department of Materials Science and Engineering at the Massachusetts Institute of Technology, USA, in 2001. After his postdoctoral fellowship in the Department of Chemistry and Chemical Biology at Harvard University, he joined Yonsei University in 2002.

Park's research has focused on self-assembled polymers and their applications to organic photoelectronics. A particular emphasis is on the development of nonvolatile memories based on ferroelectric polymers, which resulted in eight patents and two technology transfers. He has published more than 130 articles and delivered more than 60 invited talks. He is the recipient of the Wiley Young Scientist Award in 2002 and the Mid-Career Researcher Academy Award in 2014, both from the Polymer Society of Korea. He also received a minister citation from Korea's Ministry of Science, ICT and Future Planning in 2015. He is an associate editor of the journal Macromolecular Research and a board member of the Journal of Applied Polymer Science and Electronic Materials Letters. He has been involved in organizing conferences, including the International Union of Materials Research Societies, the Canadian Society for Chemistry, and NANO KOREA.

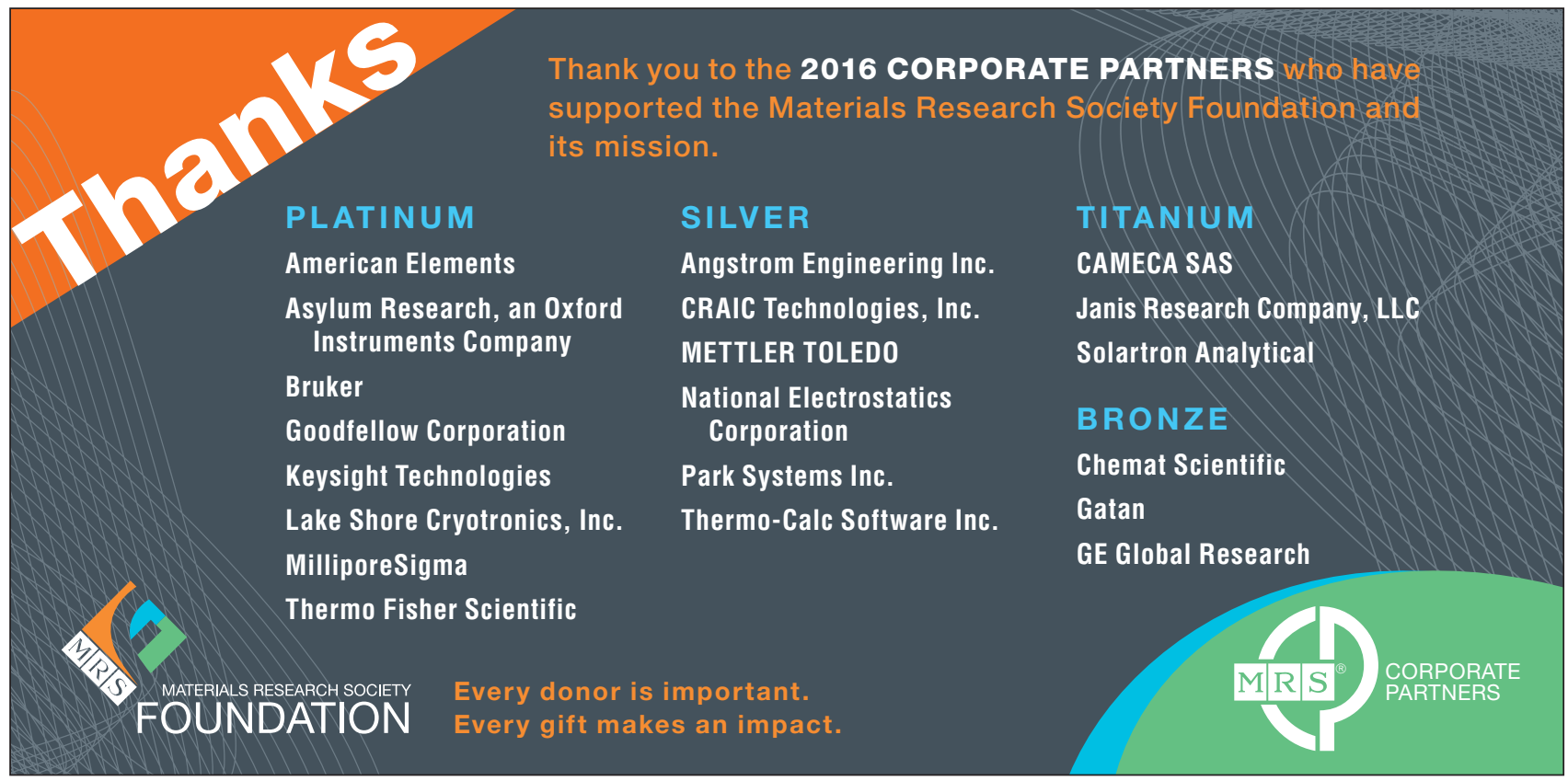

